\title{
Dissemination of Research Results: On the Path to Practice Change
}

\author{
David J Edwards
}

\section{INTRODUCTION}

$\mathrm{A}$ $s$ the medication experts in the health care system, pharmacists manage the medication needs of individual patients. Engaging in research, however, offers the opportunity not only to improve the health of those individuals encountered in daily practice but also to make a significant impact on the lives of patients across the country and internationally through the translation of research into clinical practice. Yet practice change cannot occur if clinicians are unaware of the research that has been performed. Hung and Duffet ${ }^{1}$ reviewed the status of residency projects conducted in Canada between 1999 and 2009. They identified a total of 518 projects, but less than one-third $(32.2 \%)$ had been published in any format, and only 107 $(20.6 \%)$ were ultimately published as full-length papers. Similar results were observed in an analysis of pharmacy residency projects conducted in the United States. ${ }^{2}$

Although there are a number of constraints associated with residency projects that lead many to be unsuitable for publication, these figures suggest that a considerable volume of hospital pharmacy research in Canada cannot be accessed by practitioners. It should be emphasized that researchers have an ethical obligation to at least attempt to disseminate their research findings. ${ }^{3}$ While it is tempting not to publish disappointing results, this can distort the literature on a particular topic. For example, clinicians, as well as the general public, are justifiably concerned when a pharmaceutical company elects not to publish the results of an unfavourable clinical trial, a practice that has led to a push for the creation of publicly available clinical trial databases. However, all who engage in research should be held to the same level of accountability, even if the research is not on the same scale as a multicentre clinical trial. The purpose of this paper is to provide readers with an overview of the most common methods by which research results are disseminated to the practice community and to introduce some of the concepts and barriers associated with knowledge translation.

\section{RESEARCH DISSEMINATION AT PROFESSIONAL CONFERENCES}

A variety of approaches are available for the dissemination of research findings, but by far the most common are publications in biomedical journals (discussed in detail later in this article) and presentations at professional meetings. The latter may take the form of either oral (platform) or poster presentations. Presenting clinical or practice research at a professional meeting offers the opportunity to disseminate research findings quickly, since the lag time between completing the research and presenting at a conference may be short. Furthermore, conferences are often attended by leaders in the field, who are more likely to be early adopters of research into practice. The poster format offers the additional advantage of facilitating a personal interaction between researcher and practitioner. Researchers can explain their project in detail and receive feedback that may be helpful for additional studies. Surveys of attendees at professional meetings indicate respondents' belief that knowledge transfer is enhanced when authors are available to explain and discuss their research findings at a poster session. ${ }^{4}$ Researchers should recognize that posters need to be visually appealing, to attract the widest audience at poster sessions. ${ }^{4,5}$ Presenters are competing with each other for the attention of conference attendees, and knowledge transfer will be greatest for those who do the best job of "selling" their research.

Although there is value to disseminating research findings at professional conferences, there are also disadvantages. Chief among them is a lack of depth when the results of a study are compressed into an abstract or a poster. ${ }^{5}$ One of the key tenets of research is that it must be reproducible, and the strict word limits imposed on abstracts preclude a detailed explanation of the methods used to conduct a research project. This limitation becomes less relevant if the research is later published as a full-length paper, but too often this does not occur. Prohaska and others ${ }^{6}$ examined the publication rates of abstracts submitted to 5 national pharmacy meetings in the United States. Of 2000 published abstracts, less 
than $20 \%$ were subsequently published in journals that could be accessed through PubMed or Google Scholar. This issue is not unique to pharmacy and has been observed in medicine as well. ${ }^{78}$

\section{PREPARATION OF A RESEARCH PAPER}

For research results to reach the widest possible audience and be available to practitioners not just today but permanently, they must be published in a journal. The gold standard is publication in a peer-reviewed journal that is indexed by the National Library of Medicine and other abstracting or indexing services, since this will ensure that anyone conducting a literature search will be able to locate the study. Novice researchers should be aware that the process of preparing a manuscript for publication begins before the research is even started. A well-written protocol will not only ensure successful review by the ethics committee but also form the basis for the introduction, background, and methods in the final paper. The following sections outline important considerations when preparing a paper for publication.

\section{Authorship and Journal Selection Guidelines}

As described by the International Committee of Medical Journal Editors, ${ }^{9}$ authors are expected to have made substantial contributions to the design or execution of the study or analysis of the data and must also be engaged in writing or editing of the manuscript. All authors must approve the final version of the paper. Conventions around the order of author names in the byline vary, but a general rule is that the first author has made the greatest contribution, with subsequent authors having made lesser contributions. The senior researcher may choose the final author position and will often serve as the corresponding author, particularly if the lead author is a student who may be moving on to a different institution.

Selecting an appropriate journal for each manuscript is important and must be done before the writing process begins. All journals have criteria with respect to the scope of research that is of interest to their readership, as well as style guidelines, and this information is typically provided online in "Instructions for Authors" ${ }^{10}$ Careful review of this information will ensure that the manuscript will not be rejected outright, for purely technical reasons, during editorial review. As an example, a researcher who has completed a study demonstrating the effectiveness of a drug in a rabbit model of disease should be aware that $C J H P$ "does not accept original research articles involving animal research". ${ }^{10}$

Ideally, the choice of journal should be based on the audience that would benefit most from learning about the research. When there are multiple journals in a field, factors such as circulation and impact factor may come into play. The impact factor measures the rate of citation of articles published in a journal over the previous 2 years, with higher values suggesting that papers appearing in the journal have more impact. Prestigious medical journals, such as the New England Journal of Medicine, have impact factors above 50, whereas most pharmacy practice journals in North America tend to have impact factors below 3, and some are not indexed in MEDLINE. ${ }^{11} \mathrm{~A}$ low impact factor does not mean that a pharmacy journal is of poor quality; rather, it may reflect the relatively small readership and the fact that most readers are not researchers and thus are less likely to subsequently write a paper citing an article that they have read.

\section{Organization of a Research Paper}

The body of the paper begins with the Introduction, which should concisely outline the rationale for conducting the research and state the purpose or hypothesis being tested. Given the large number of papers that practitioners must review to stay current, the potential value of the research to practice must be made evident to the reader as early as possible.

The Methods section should present an overview of how the research was conducted, in sufficient detail that others could duplicate the work. Chan and Altman ${ }^{12}$ reviewed over 500 clinical trials and reported that fewer than half provided adequate descriptions of items such as sample size calculation, primary outcomes, method of randomization, and handling of attrition. This problem can be largely avoided through the use of reporting guidelines, which provide detailed recommendations and checklists. For example, the EQUATOR (Enhancing the QUAlity and Transparency Of health Research) network has compiled 276 reporting guidelines covering a wide range of study designs used in clinical, preclinical, epidemiologic, and cost-effectiveness research. ${ }^{13}$ For pharmacokinetic studies, Kanji and others ${ }^{14}$ have recently developed a comprehensive checklist of 24 items that should be reported.

The Results section presents the key findings of the research without commentary or discussion, through a combination of text, tables, and figures. A well-designed figure can convey complex research data in a way that readers will find much more understandable than if the same information were presented in the text, but a figure should not duplicate data presented in the text or tables. Importantly, the results should match the methods. In other words, there should be results for all of the procedures listed in the Methods, and the reader should not find results for which no methods are described.

The Discussion typically includes a summary of the major research findings, an assessment of the importance of the results in the context of previously published studies, the limitations of the study, and suggestions for future research. The conclusions may be part of the Discussion or a separate section, depending on journal policy, and should address the original hypothesis or purpose. Authors should provide a balanced and unbiased evaluation of the validity and value of the results. Contradictory findings from previous studies should be addressed, not ignored. In addition, care should be taken to ensure that the conclusions 
are not overstated. Although it is tempting to speculate about the broad applicability of the findings, the external validity of studies with highly controlled conditions and extensive inclusion and exclusion criteria may be limited.

\section{The Writing Process}

It can be difficult for many researchers, particularly those who are also practitioners, to find an extended block of time to write. This challenge is often cited as the primary reason why data remain in file cabinets and manuscripts go unwritten. If the thought of preparing a 3000-word paper is intimidating, consider breaking it up into smaller chunks that can be written in short blocks of time. A single double-spaced page can be written in 1 to 2 hours, and writing for this period of time every day or two should produce a completed first draft of a manuscript in only a few weeks. Given the time constraints that every clinicianresearcher faces, spending hours trying to craft the perfect sentence is a luxury that cannot be afforded. Writers should keep in mind that first drafts will not be widely distributed, and it is important to get something down on paper, even if it only roughly expresses the point to be made.

Health care professionals usually receive little or no formal training in writing. It is therefore important to find a mentor who not only is willing to take the time to read early drafts but also can be trusted to provide constructive criticism. Authors must learn to be receptive to suggestions and not defensive about their writing. Other health care professionals who are not pharmacists may also provide valuable advice. Because they will typically not be "content experts", they may be able to provide a more unbiased critique with respect to the paper's readability. The use of jargon and acronyms that are in widespread use in pharmacy should be avoided, particularly if the potential audience will include individuals with a wide range of backgrounds.

\section{The Peer Review Process}

Once a manuscript has been submitted to the journal of choice, it undergoes an initial editorial review to ensure that the paper meets expected standards for quality and style and that the content fits with the journal's scope. Peer reviewers are then identified who have the expertise to provide an unbiased assessment of the quality and importance of the research.

The peer review process is not without fault. It is an unpaid activity that reviewers volunteer to undertake out of a sense of professional duty. However, not all reviewers have the appropriate expertise or take the time to conduct a thorough review. As discussed by Walker and Rocha da Silva, ${ }^{15}$ problems related to peer review can include failure to recognize the importance of truly novel discoveries, lack of consensus among reviewers, failure to detect serious errors in study design, and unethical practices by some reviewers, who may deliberately delay publication, reject the work of competitors in their field, or even steal ideas for their own research. Research that fails to show a difference in the hypothesized outcome is more likely to be rejected and remain unpublished, a phenomenon referred to as "bias against the null hypothesis". Koren and others ${ }^{16}$ reported that research indicating that a drug is teratogenic is more likely to be published and publicized than studies demonstrating no damaging effect on the fetus.

Attempts to improve peer review have included making papers available online before publication to allow for open commentary from the scientific community. Bias during peer review may be minimized by masking the identity of the authors of manuscripts or by creating a transparent process whereby the identities of both authors and reviewers are known to each other. This approach is meant to discourage anonymous reviewers from providing harsh and unsubstantiated criticism that they would be unlikely to write if their identity were known to the authors.

Despite these problems with peer review, it continues to be the cornerstone of the review process in professional journals, and most reviews supply helpful comments that will ultimately improve a paper. Authors must address, but need not necessarily agree with, all of the reviewers' comments, and inexperienced authors should be aware that rejection of a manuscript does not mean that the research has no value. A number of important papers that have resulted in significant knowledge translation were not accepted by the first journal to which they were submitted.

\section{KNOWLEDGE TRANSLATION}

Researchers should not expect that practice change will inevitably occur once their research results are published. A commonly quoted estimate is that it takes an average of 17 years for $14 \%$ of published evidence to be widely incorporated into clinical practice. ${ }^{17}$ Efforts to close this gap between evidence and practice are referred to as "knowledge translation", a term defined by the Canadian Institutes of Health Research as a "dynamic and iterative process that includes the synthesis, dissemination, exchange and ethically sound application of knowledge to improve health, provide more effective health services and products, and strengthen the health care system". ${ }^{18}$ As discussed in a recent editorial in $C J H P,{ }^{19}$ knowledge translation is a complex process that is subject to multiple barriers, including factors such as the personality characteristics and culture of the members of a profession. ${ }^{20}$

Researchers have historically considered their work to be complete once a paper based on their research has been presented and published. However, it is clear that this passive, unfocused approach, referred to as diffusion, ${ }^{21}$ is often ineffective in changing practice. More active efforts to increase the exchange of knowledge between researcher and clinician are termed "dissemination" and may include the development of targeted messages for professional organizations or other stakeholders, the establishment of practice guidelines, and the use of knowledge brokers. ${ }^{21}$ The latter are 
individuals who possess communication skills, clinical experience, and scientific expertise, specifically hired by organizations to improve practice by bridging the gap between researchers and clinicians. ${ }^{21,22}$

Efforts by researchers to actively disseminate knowledge to targeted audiences are also referred to as a "push" process. ${ }^{21}$ An example is the report by Truong and others, ${ }^{23}$ who prepared a letter summarizing the results of the SCRIP-HTN trial in which an intervention by a pharmacist-nurse team was able to produce better clinical outcomes in patients with diabetes mellitus and hypertension. This communication was forwarded to 22 professional pharmacy organizations in Canada but, unfortunately, only 3 used this information to engage in any knowledge translation activities over the following 6-month period. A significant barrier was that many of these organizations did not view knowledge translation as a priority or as part of their mandate. Clinicians and policy-makers can contribute to knowledge translation using a "pull" approach, whereby evidence from the literature is used to prepare a systematic review or develop practice guidelines or is disseminated throughout a practice network..$^{21}$

\section{CONCLUSION}

The dissemination of research is an important first step on the path toward knowledge translation and practice change. Presenting research at professional meetings allows for more rapid dissemination of research findings, but the audience may be narrow, and the depth of information that can be provided in this format is limited. Pharmacists engaging in clinical or practice research should pursue the publication of their research in peerreviewed journals, to ensure that the information is permanently available to the widest possible audience. Where appropriate, researchers are also encouraged to develop targeted messages for key stakeholders regarding their research, to enhance knowledge translation, and knowledge users can facilitate this process through systematic reviews, guideline development, and communication through practice networks.

\section{References}

1. Hung M, Duffett M. Canadian pharmacy practice residents' projects: publication rates and study characteristics. Can J Hosp Pharm. 2013; 66(2):86-95.

2. McKelvey RP, Hatton RC, Kimberlin CA. Pharmacy resident project publication rates and study designs from 1981, 1991, and 2001. Am J Health Syst Pharm. 2010;67(10):830-6.

3. Pearn J. Publication: an ethical imperative. BMJ. 1995;310(6990):1313-5.

4. Rowe N, Ilic D. What impact do posters have on academic knowledge transfer? A pilot survey on author attitudes and experiences. BMC Med Educ. 2009;9:71.

5. Rowe N, Ilic D. Poster presentation - a visual medium for academic and scientific meetings. Paediatr Respir Rev. 2011;12(3):208-13.
6. Prohaska E, Generali J, Zak K, Grauer D. Publication rates of abstracts presented at five national pharmacy meetings. Hosp Pharm. 2013;48(3): 219-26.

7. Amirhamzeh D, Moor MA, Baldwin K, Hosalkar HS. Publication rates of abstracts presented at Pediatric Orthopaedic Society of North America meetings between 2002 and 2006. J Pediatr Orthop. 2012;32(2):e6-e10.

8. Kinsella SD, Menge TJ, Anderson AF, Spindler KP. Publication rates of podium versus poster presentations at the American Orthopaedic Society for Sports Medicine meetings: 2006-2010. Am J Sports Med. 2015;43(5): $1255-9$

9. Defining the role of authors and contributors. International Committee of Medical Journal Editors; [cited 2015 Jun 29]. Available from: www.icmje.org/ recommendations/browse/roles-and-responsibilities/defining-the-role-ofauthors-and-contributors.html

10. Canadian Journal of Hospital Pharmacy. Information for authors [website]. Ottawa (ON): Canadian Society of Hospital Pharmacists; [cited 2015 Jun 29]. Available from: www.cjhp-online.ca/index.php/cjhp/pages/view/authors

11. Journal impact factor list. International Scientific Institute; [cited 2015 Jul 10]. Available from: www.scijournal.org/

12. Chan AW, Altman DG. Epidemiology and reporting of randomised trials published in PubMed journals. Lancet. 2005;365(9465):1159-65.

13. Library for health research reporting. Equator Network; [cited 2015 Jun 30]. Available from: www.equator-network.org/library/

14. Kanji S, Hayes M, Lind A, Shamseer L, Chant C, Edwards DJ, et al. Reporting guidelines for clinical pharmacokinetic studies: the ClinPK statement. Clin Pharmacokinet. 2015;54(7):783-95.

15. Walker R, Rocha da Silva P. Emerging trends in peer review_-a survey. Front Neurosci. 2015;9:169.

16. Koren G, Madjunkova S, Maltepe C. Bias against the null hypothesis: scaring pregnant women about drugs in pregnancy. Can Fam Physician. 2014;60(5):441-2.

17. Westfall JM, Mold J, Fagnan L. Practice-based research—-blue highways" on the NIH roadmap. JAMA. 2007;297(4):403-6.

18. Strauss SE, Tetroe J, Graham I. Defining knowledge translation. CMAJ. 2009;181(3-4):165-8.

19. Simpson SH. Applying new evidence into practice: a need for knowledge translation. Can J Hosp Pharm. 2015;68(2):100-1.

20. Rosenthal M, Hall KW, Bussières JF, Tsuyuki RT. Professional culture and personality traits of hospital pharmacists across Canada: a fundamental first step in developing effective knowledge translation strategies. Can J Hosp Pharm. 2015;68(2):127-35.

21. Gagnon ML. Moving knowledge to action through dissemination and exchange. J Clin Epidemiol. 2011;64(1):25-31.

22. Hoens AM, Reid WD, Camp PG. Knowledge brokering: an innovative model for supporting evidence-informed practice in respiratory care. Can Respir J. 2013;20(4):271-4.

23. Truong K, Rosenthal M, Tsuyuki RT. Asleep at the wheel: pharmacy practice research advocacy and knowledge translation by Canadian pharmacy organizations. Can Pharm J. 2010;143(2):78-81.e1.

David J Edwards, BSCPharm, PharmD, MPH, FCCP, is Hallman Director and Professor at the School of Pharmacy, University of Waterloo, Waterloo, Ontario.

Competing interests: None declared.

\section{Address correspondence to:}

Dr David J Edwards

School of Pharmacy

University of Waterloo

200 University Avenue West

Waterloo ON N2L $3 G 1$

e-mail: david.edwards@uwaterloo.ca

For a complete list of articles in the Research Primer series, see page 469. 
This is the 15th and final article in the CJHP Research Primer Series, an initiative of the CJHP Editorial Board and the CSHP Research Committee. The 2-year series was designed to appeal to relatively inexperienced researchers, with the goal of building research capacity among practising pharmacists. The articles, presenting simple but rigorous guidance to encourage and support novice researchers, were solicited from authors with appropriate expertise.

Complete list of articles in this series:

Bond CM. The research jigsaw: how to get started. Can J Hosp Pharm. 2014;67(1):28-30.

Tully MP. Research: articulating questions, generating hypotheses, and choosing study designs. Can J Hosp Pharm. 2014;67(1):31-4.

Loewen P. Ethical issues in pharmacy practice research: an introductory guide. Can J Hosp Pharm. 2014;67(2):133-7.

Tsuyuki RT. Designing pharmacy practice research trials. Can J Hosp Pharm. 2014;67(3):226-9.

Bresee LC. An introduction to developing surveys for pharmacy practice research. Can J Hosp Pharm. 2014;67(4):286-91.

Gamble JM. An introduction to the fundamentals of cohort and case-control studies. Can J Hosp Pharm. 2014;67(5):366-72.
Austin Z, Sutton J. Qualitative research: getting started. Can J Hosp Pharm. 2014;67(6):436-40.

Houle S. An introduction to the fundamentals of randomized controlled trials in pharmacy research. Can J Hosp Pharm. 2015; 68(1):28-32.

Charrois TL. Systematic reviews: What do you need to know to get started? Can J Hosp Pharm. 2015;68(2):144-8.

Sutton J, Austin Z. Qualitative research: data collection, analysis, and management. Can J Hosp Pharm. 2015;68(3):226-31.

Cadarette SM, Wong L. An introduction to health care administrative data. Can J Hosp Pharm. 2015;68(3):232-7.

Simpson SH. Creating a data analysis plan: what to consider when choosing statistics for a study. Can J Hosp Pharm. 2015;68(4):311-7.

Dolovich L. Playing in the sandbox: considerations when leading or participating on a multidisciplinary research team. Can J Hosp Pharm. 2015;68(5):401-5.

Kanji S. Turning your research idea into a proposal worth funding. Can J Hosp Pharm. 2015;68(6):458-64.

Edwards DJ. Dissemination of research results: on the path to practice change. Can J Hosp Pharm. 2015;68(6):465-9.

\section{CJHP Subscriptions 2016 / Abonnements au JCPH 2016}

In 2015, we moved to an online only version of CJHP and introduced the institutional online subscription option. CJHP online is included as a benefit of CSHP membership. All prices are in Canadian funds.

En 2015, nous avons migré vers la publication d'une seule version du JCPH, soit la copie électronique en ligne, et nous avons introduit en option un abonnement institutionnel. L'abonnement à la version électronique du $J C P H$ publiée en ligne est inclus dans les droits d'inscription à la SCPH. Tous les prix sont en dollars canadiens.

\begin{tabular}{|c|c|c|}
\hline $\begin{array}{l}\text { Subscriber group / } \\
\text { Groupe d'abonnés }\end{array}$ & $\begin{array}{l}\text { Individual Online Subscription / } \\
\text { Abonnement individuel en ligne }\end{array}$ & $\begin{array}{l}\text { Institutional Online Subscriptions / } \\
\text { Abonnement institutionnel en ligne }\end{array}$ \\
\hline $\begin{array}{l}\text { Nonmembers / } \\
\text { Non-membres }\end{array}$ & $\begin{array}{l}\$ 160.00 \text { per year, plus GST or HST } \\
160,00 \$ \text { par an, plus TPS ou TVH }\end{array}$ & $\begin{array}{l}\$ 480.00 \text { per year, plus GST or HST } \\
480,00 \$ \text { par an, plus TPS ou TVH }\end{array}$ \\
\hline
\end{tabular}

If you would like to purchase a subscription, please fill-out our CJHP 2016 Subscription Application Form, which can be found on the CJHP website: www.cjhp-online.ca. Please direct any comments or questions to cjhpedit@cshp.ca.

Si vous désirez vous abonner, veuillez remplir le formulaire d’abonnement au JCPH 2016. Vous pouvez I'obtenir en visitant le site Web du JCPH : www.cjhp-online.ca. Pour tout commentaire ou toute question, veuillez vous adresser à cjhpedit@cshp.ca. 